\title{
Use of the Remote Food Photography Method by young pregnant women: a feasibility study
}

\author{
C.Y. England ${ }^{1,3}$, D.A. Lawlor ${ }^{2}$, M. Lewcock ${ }^{2}$, I. Craddock ${ }^{4}$, A. Skinner ${ }^{4}$, C.K. Martin ${ }^{5}$ and \\ L. Johnson ${ }^{1,3}$ \\ ${ }^{1}$ Centre for Exercise, Nutrition and Health Science, School for Policy Studies University of Bristol, BS8 1TZ, UK, \\ ${ }^{2}$ Medical Research Council Integrative Epidemiology Unit, University of Bristol, UK, BS8 2BN, ${ }^{3}$ NIHR Bristol \\ Biomedical Research Unit in Nutrition, University of Bristol, UK, BS2 8AE, ${ }^{4}$ Faculty of Engineering, University of \\ Bristol, BS8 IUB, UK and ${ }^{5}$ Pennington Biomedical Research Centre, Louisiana State University, Baton Rouge, United \\ States of America
}

Pregnancy is considered an underutilised opportunity for promoting lifestyle changes that have health benefits for a woman and her developing child. Changes to maternal diet during pregnancy have, to date, been assessed primarily by food frequency questionnaires which may be inaccurate. We have tested the feasibility of using a validated method of dietary data capture, using a smartphone application (app), in young (aged 23-25), UK-based, pregnant women.

The Remote Food Photography Method (RFPM) collects dietary data using the SmartIntake app ${ }^{(1,2)}$. This method has been previously validated against doubly labelled water in 50 free-living US adults and underestimated energy intake by only $3 \cdot 7 \%{ }^{(1)}$. Our study fieldworkers were trained in use of the app and practiced capturing dietary data themselves for at least 2 full days prior to training participants. Women were asked to record 6 full days of dietary intake by taking a photo before and after each eating/drinking occasion, and providing a brief text description of items they thought might be hard to identify from the image. The photos were sent, via e-mail, to a server for analysis by experts. Automatic reminder e-mails, at times of the participant's choosing, were generated and sent to the smartphone. Real-time monitoring of the quality of the photos and feedback to participants occurred for the first day. Data collection began in October 2015 and is on-going. We present usability findings from the first 6 months.

Seventy-three participants were invited to use SmartIntake of which 25 (34\%) agreed, with one participant withdrawing consent before capturing any photos. Fieldworkers estimated that training took around 20 minutes. Most participants found installation and set-up $(95 \%)$, taking photos of meals $(70 \%)$ and receiving reminders $(81 \%)$ easy or very easy. Twenty-one $(84 \%)$ provided enough dietary data for analysis. The median number of days on which participants used the app was 6 (IQR 4-6): 14 (58\%) women recorded at least one eating/drinking occasion on 6 days, although $4(17 \%)$ used the app on 3 days or fewer. The women captured a total of 484 eating/drinking occasions; $66 \%$ of these included a text description. The total median number of photos taken/person was 42 (IQR 19-61) with a median 7 (IQR 5-11) photos/day. The maximum number of photos taken by a single participant was 94 . 75\% of eating/ drinking occasions were captured in 2 photos, with only $41(8 \%)$ occasions taking 4 or more photos. The maximum number of photos for one meal was 12 (starter, main course, pudding, drinks).

Fieldworkers reported that the main reasons for refusing to use the app were that the women were unable to use a phone at work or were too busy. Of the participants who provided fewer than 4 days, three reported that it was too difficult due to family commitments and one gave no reason. Nine other women provided comments on use; all found data capture inconvenient but 10 women (out of 18 who answered) indicated that they would use the method again.

These results show that the phone app is feasible for use in some pregnant women but the ability to use this method is influenced by family and work commitments and the method may be burdensome, especially when meals are complex.

This work is supported by the Elizabeth Blackwell Institute for Health Research. The UK Medical Research Council, Wellcome Trust and University of Bristol support data collection in this pregnancy cohort.

1. Martin CK, Correa JB, Han H et al. (2012) Obesity 20, 891-9.

2. Martin CK, Nicklas T, Gunturk B et al. (2014) J Hum Nutr Diet. 27, 72-81. 